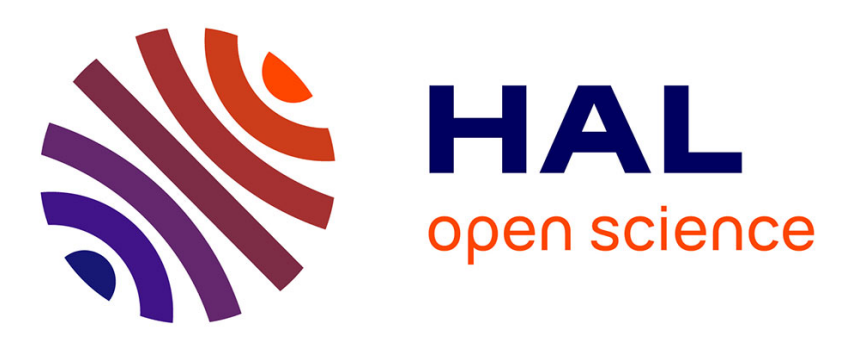

\title{
A Multiscale Cardiac Model for Fast Personalisation and Exploitation
}

\author{
Roch Molléro, Xavier Pennec, Hervé Delingette, Nicholas Ayache, Maxime
}

Sermesant

\section{- To cite this version:}

Roch Molléro, Xavier Pennec, Hervé Delingette, Nicholas Ayache, Maxime Sermesant. A Multiscale Cardiac Model for Fast Personalisation and Exploitation. Medical Image Computing and Computer Assisted Intervention (MICCAI), Oct 2016, Athens, Greece. pp.174-182, 10.1007/978-3-319-467269_21. hal-01360908

\section{HAL Id: hal-01360908 https://hal.inria.fr/hal-01360908}

Submitted on 6 Sep 2016

HAL is a multi-disciplinary open access archive for the deposit and dissemination of scientific research documents, whether they are published or not. The documents may come from teaching and research institutions in France or abroad, or from public or private research centers.
L'archive ouverte pluridisciplinaire HAL, est destinée au dépôt et à la diffusion de documents scientifiques de niveau recherche, publiés ou non, émanant des établissements d'enseignement et de recherche français ou étrangers, des laboratoires publics ou privés. 


\title{
A Multiscale Cardiac Model for Fast Personalisation and Exploitation
}

\author{
Roch Mollero ${ }^{1}$, Xavier Pennec ${ }^{1}$, Hervé Delingette ${ }^{1}$, Nicholas Ayache ${ }^{1}$, and \\ Maxime Sermesant ${ }^{1}$ \\ INRIA - Asclepios Research Project, Sophia Antipolis, France
}

\begin{abstract}
Computer models of the heart are of increasing interest for clinical applications due to their discriminative and predictive abilities. However a single 3D simulation can be computationally expensive and long, which can make some practical applications such as the personalisation phase, or a sensitivity analysis of mechanical parameters over the simulated behaviour quite slow. In this manuscript we present a multiscale 0D/3D model which allows us to have a reliable (and extremely fast) approximation of the behaviour of the 3D model under a few simplifying assumptions. We first detail the two different models, then explain the coupling of the two models to get fast $0 \mathrm{D}$ approximation of $3 \mathrm{D}$ simulations. Finally we demonstrated how the multiscale model can speed-up an efficient optimization algorithm, which enables a fast personalisation of the $3 \mathrm{D}$ simulations by leveraging on the advantages of each scale.
\end{abstract}

\section{Introduction}

Three-D personalised cardiac models simulate the physical behaviour of a patient heart, in order to perform advanced analysis of the cardiac function. The movement of the myocardium is calculated under the influence of simulated electromechanics and haemodynamic condition, which requires complex and expensive calculations, particularly as the spatial and temporal resolution of the model increases (1 hour per heart beat, even several days for some models).

This computational burden particularly impacts applications of the models where many simulations are required. The model personalisation phase (fitting the available clinical data) is particularly impacted, slowing the study of clinical applications such as disease caracterisation and prediction of case evolution

To tackle this computational burden, surrogate models of the cardiac function have been developed, for example for uncertainty quantification [1] or probability estimation [2] over the cardiac parameters. Those models usually rely on regression models which require many pre-computations to approximate nonlinear behaviours, thus scaling badly as the number of varying parameters increases.

Here we built a multiscale cardiac model by developping an novel coupling method between an original 3D cardiac model and a reduced lumped "0D" version of the same model. While the coupling of different scales has been used to improve boundary conditions in cardiac modelling and computational fluid dynamics [3], we propose here the simultaneous simulation of the same model at 
different scales, which enables extremely fast approximations of the behaviour of the 3D model with 0D simulations. It provides a natural approximation of all the desired cardiac values. Furthermore, we demonstrate the efficiency of the multiscale $0 \mathrm{D} / 3 \mathrm{D}$ model when plugged into a personalisation process driven by the genetic algorithm CMA-ES. This creates a very fast and computationally efficient personalisation agent, successfully tested on the personalisation of 34 hearts with different geometries.

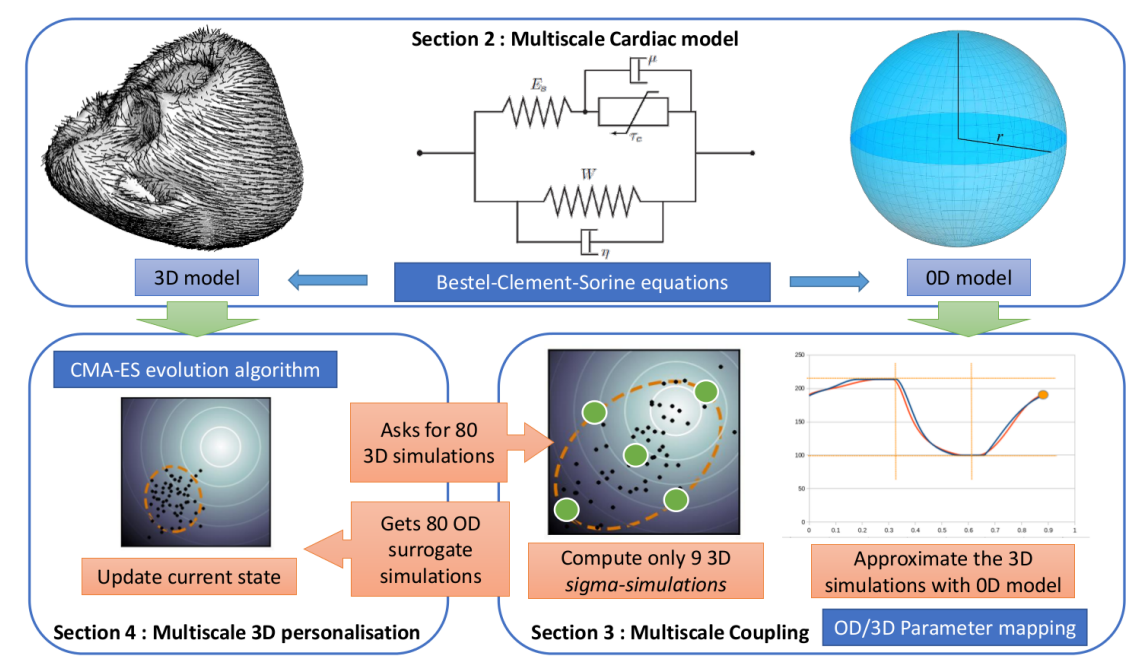

Fig. 1: Multiscale model and personalisation pipeline

\section{Multiscale Cardiac Model}

In order to build a computer model of the myocardium, one needs different components to represent the passive stiffness, the active contraction and the boundary conditions (hemodynamics). We use here the Bestel-Clement-Sorine model (BCS) of the sarcomere contraction, in conjonction with the MooneyRivlin model for passive elasticity as described in [4]. Hemodynamics are represented through global values of pressures and flows in the cardiac chambers.

\subsection{Three-D Cardiac Model}

Here we first use 3D biventricular mesh geometry extracted from MRI images, with synthetic myocardial fibres. The active sarcomere force and the passive force are computed at each point of the mesh with the Bestel-Clement-Sorine (BCS) model under the influence of the pre-computed electrophysiology. The boundary condition is a circulation model with the 4 phases of the cardiac cycle based using a 3-parameters Windkessel model of blood pressure as after-load. We also specify a resting mesh in addition of the initial mesh geometry to start the simulation with a geometry which already includes residual stress. 
The complete details of the 3D electromechanical model as well as the $\mathrm{C}++$ implementation of the finite element solver are discussed in [5]. With meshes of 10 000 and 15000 points and a time step of $5 \mathrm{~ms}$, a single beat (around $0.9 \mathrm{~s}$ ) takes between 25 minutes and 1 hour to compute. Finally, since we want to consider cardiac beats which are not affected by the initialization of the simulation, we compute 3 subsequent beats per simulation and only consider the last one, so the full $3 \mathrm{D}$ simulation takes between 75 minutes and 3 hours to be computed.

\subsection{D Cardiac Model}

If we then make simplifying assumptions on the geometry and on the properties of the material, by approximating the ventricular shape as a sphere, we can derive the equations of a fast lumped model of the heart as detailed in [6].

The myocardial motion is then only described by the inner radius $\mathbf{r}$ of the ventricle (the "Resting Mesh" in 3D becomes simply a "Resting Radius" r0), the material is considered incompressible and has idealised tangential fibres. The electrophysiological activation is supposed synchronous and homogeneous over the sphere as well as the resulting active sarcomere force. Finally we use the same circulation model and Mooney Rivlin equations than in the 3D model.

We implemented the 0D model within the CellML framework, then converted into $\mathrm{C}$ code with OpenCOR [7]. With a temporal discretisation of 0.1 milliseconds, we can simulate 10 to 20 heart beats per seconds.

\section{Multiscale Coupling}

Although with different mechanical assumptions, both models follow the same trends in behaviour when we change the global parameters. The idea of the coupling is to have those trends match within a specific domain of 3D parameters, in order to predict the behaviour of many 3D simulations by only computing 0D simulations. This is done by first matching several 3D simulations of the domain (called the sigma-simulations) with the 0D model, then learning a mapping between the $3 \mathrm{D}$ parameters and the $0 \mathrm{D}$ parameters.

\subsection{Personalisation of the 0D Model to a 3D Simulation}

The first step of the coupling is to make the two models match on a single simulated beat. Given one 3D simulated heartbeat, we want to accurately reproduce the pump behaviour of the heart with the $0 \mathrm{D}$ model (under the same boundary conditions). In particular we focus on reproducing 6 features of the volume curve by setting 5 main electromechanical parameters in the $0 \mathrm{D}$ model, both listed in Table 1.

We estimate the parameters by performing a derivative-free optimisation with the genetic algorithm CMA-ES [8]. This powerful algorithm combines Bayesian

principles of Maximum Likelihood with a local gradient descent on the members of each generation, by updating at each iteration both an internal covariance Ic 
Table 1: Features and parameters in the 0D model personalisation over a $3 \mathrm{D}$ simulation

\begin{tabular}{l|l}
\hline Features & 0D Parameters \\
\hline Maximal Volume Vmax & Maximal Contraction $\sigma$ \\
Minimal Volume Vmin & Stiffness $\mathbf{c 1}$ \\
Percentage of filling when the atrium contracts $\mathbf{F V a t}$ & Resting radius $\mathbf{r} \mathbf{0}$ \\
Flow when the atrium contracts Qat & damping eta \\
Time where the volume is minimum $\mathbf{t V m i n}$ & Atrio-Ventricular Delay $\mathbf{A V}$. \\
Time when ejection starts teject & \\
\hline
\end{tabular}

and mean Im of the parameters to explore at the next iteration. We define the fitness (score) of each simulation by:

$$
\text { fitness }(x)=\|(\text { Values }(x)-\text { TargetValues }) / \text { Normalization } \|
$$

which is the $L_{2}$ distance between the simulated and the objective values, normalized to be able to compare volumes (unit_volume $=10 \mathrm{ml}$ ) with times (unit_time $=20 \mathrm{~ms}$ ), percentages (unit_percent $=5 \%$ ) and flow (unit_flow $=10 \mathrm{ml} / \mathrm{s}$ ).

We use a population of 20 simulations per generation, and optimize over 100 generations. This takes less than 10 minutes on a multi-core computer with parallel computation of the simulations within each generation. Figure 3 shows the matching behaviours of a $3 \mathrm{D}$ simulation and its $0 \mathrm{D}$ counterpart.

\subsection{Prediction of 3D behaviour from 0D Simulations}

We want to be able to get reliable approximations of other 3D simulations within a domain $\Omega_{3 D}$ of 3D parameters by doing $0 \mathrm{D}$ simulations, so we need to be able to convert a vector $X \in \Omega_{3 D}$ into a relevant vector $\mathrm{Y}$ of $0 \mathrm{D}$ parameters which best approximates the $3 \mathrm{D}$ simulations. Here we focus in the mapping of $N_{1}=4$ $3 \mathrm{D}$ parameters toward $N_{2}=50 \mathrm{D}$ parameters listed in Table 2 .

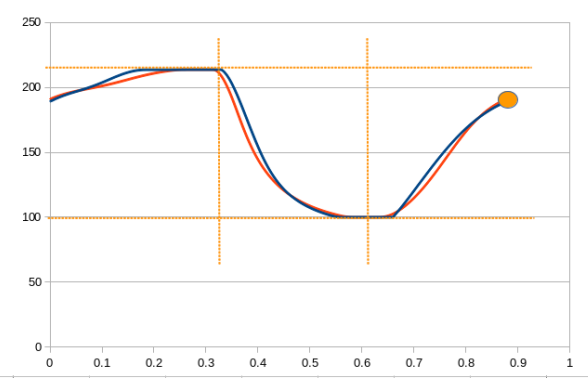

LV Volume (ml) over time $\mathrm{t}(\mathrm{s})$

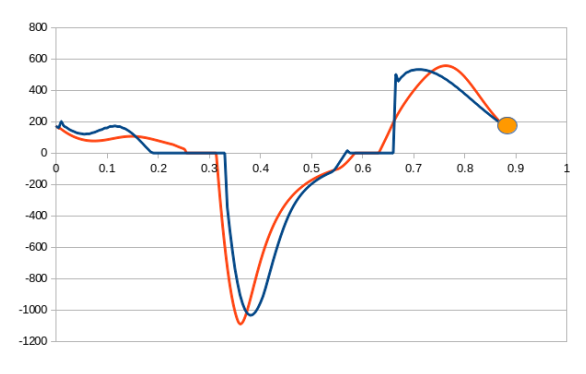

LV Flow $(\mathrm{ml} / \mathrm{s})$ over time $\mathrm{t}(\mathrm{s})$

Fig. 3: Comparison between a volume curve simulated with the 3D model (blue) and the matched $0 \mathrm{D}$ simulation (red). Orange lines and points outline the 6 fitted values 
The parameters do not behave necessarily the same for both models (and not in the same range of values). For example the 3D and 0D dampings are not calculated in the same way, and the "resting position" variable is a unitless value from 0 to 10 in the 3D case, whereas it is the "inner radius" (in $\mathrm{cm}$ ) in the $0 \mathrm{D}$ mdoel. Also even for parameters coming from the same equations in both models (such as $\sigma$ and $\mathbf{c 1}$ ) the values might be different for two matching 0D and 3D simulations. For example we can get the same volume curve with contractility and stiffness values which are 10 times smaller in the 0D implementation than in the $3 \mathrm{D}$ setting). The mapping is thus not trivial and is done through the following steps:

1. We first perform $2 N_{1}+13 \mathrm{D}$ simulations at 2 points of each of the $N_{1}$ principal directions of the domain of interest $\Omega_{3 D}$ and its center (called the sigma-simulations with parameters $S_{i}$ ).

2. We then fit the 0D model on each of those $2 N_{1}+1$ simulations with the method explained in the previous subsection, which gives the $2 N_{1}+1$ corresponding vectors of $0 \mathrm{D}$ parameters $V_{i}$.

3. Then we compute a least square linear regression $\phi$ between the parameters of $S_{i}$ and the parameters $V_{i}$.

4. Finally for each vector $\mathrm{X}$ of $\Omega_{3 D}$, we get the relevant vector $Y=\phi(X)$ of 0D parameters by using this linear regression.

\subsection{Results of the Parameter Mapping}

Here we evaluate the approximation of 80 other $3 \mathrm{D}$ simulations with parameters $X_{j} \in \Omega_{3 D}$ by their corresponding 0D simulations with parameters $Y_{j}=\phi\left(X_{j}\right)$. We focus on the error made on 7 features, which we compare with the error made by a direct linear regression between the parameters of the sigma-simulations and the 7 features (which is the best regression model we can run with this small number of points). Results are shown in Table 3.

Both methods provide good approximations of the features. However if we exclude $\mathbf{t V m i n}$ (which is harder to approximate because of the temporal discretisation of the models), the simulations with the $0 \mathrm{D}$ model provide overall better approximations of the values, particularly of those with the most nonlinear behaviour such as the percentage of filling (FVat) and the flow (Qat) when the atrium opens (resp by $43 \%$ and $40 \%$ ). This is because the 0D model

Table 2: Three-D and 0D Parameters of the Mapping

\begin{tabular}{l|l}
\hline Three-D Parameters & OD Parameters \\
\hline Maximal Contraction $\sigma$ & Maximal Contraction $\sigma$ \\
Stiffness $\mathbf{c 1}$ & Stiffness $\mathbf{c 1}$ \\
Resting Mesh $\mathbf{R M}$ & Resting radius $\mathbf{r} \mathbf{0}$ \\
$3 D$ damping $\mu$ & OD damping eta \\
& Atrio-Ventricular Delay $\mathbf{A V}$ \\
\hline
\end{tabular}


Table 3: Feature approximation by the Mapping: Range of values of each feature, mean absolute error with the mapping $\left(M A E_{m}\right)$ and mean absolute error with the direct regression $\left(M A E_{r}\right)$

\begin{tabular}{|c|c|c|c|c|c|c|c|}
\hline Feature & $\mathrm{tVmin}(\mathrm{ms})$ & $\mathrm{V} \max (\mathrm{ml})$ & $\operatorname{Vmin}(\mathrm{ml})$ & $\mathrm{EF}(\%)$ & $\mathrm{SV}(\mathrm{ml})$ & FVat $(\%)$ & Qat $(\mathrm{ml} / \mathrm{s})$ \\
\hline Range & 30.3 & 9.97 & 34.6 & 27.7 & 29.7 & 11.1 & 93.9 \\
\hline$M A E_{m}$ & 6.35 & 2.03 & 1.06 & 0.17 & 0.98 & 0.78 & 3.62 \\
\hline$M A E_{r}$ & 3.86 & 2.09 & 0.89 & 0.15 & 1.21 & 1.38 & 5.99 \\
\hline
\end{tabular}

naturally captures the nonlinear behaviour of those features which would require many simulations to be well approximated by a nonlinear regression model.

\section{Efficient Personalisation of the Multiscale Model}

Personalisation is the first step for the application of 3D cardiac modelling to patient-specific data. After extracting a patient-specific mesh geometry from the images, we want the 3D simulation to match specific clinical data. This can be done as in Section 3.1, by optimizing some parameters of the 3D cardiac model with CMA-ES to minimize a score related to the distance between the simulation and the target clinical data.

Since the convergence speed of CMA-ES increases with the size of the population evaluated at each iteration, we want to set this size as high as possible, but the computational cost of each 3D simulation is the limiting factor. However with the coupled model, we show that we can evaluate the scores of the members of a large population with a limited number of 3D simulations, which allows to benefit from the increased speed of convergence while keeping a reasonable computational burden.

\subsection{Full Personalisation Pipeline}

In the personalisation process of the 3D model, we want to reproduce 5 features of the volume curve (listed in Table 4 ) by optimizing the 4 parameters described in Section 3.2. We initialize a CMA-ES optimization process with a high population size $\mathrm{m}=80$, and derive the Coupled-CMA optimization method by adapting the original CMA-ES as follows:

At each iteration, the CMA-ES algorithm asks for the scores of $\mathrm{m} 3 \mathrm{D}$ simulations $X_{j}$ whose parameters are drawn from a multivariate distribution, ruled by its own internal mean $\mathbf{I m}$ and covariance $\mathbf{I c}$.

Instead of performing these $\mathrm{m}$ 3D simulations, we calculate a mapping $\phi$ and $\mathrm{m}$ surrogate $0 \mathrm{D}$ simulations with parameters $Y_{j}=\phi\left(X_{j}\right)$, as explained in 3.2. Only the $2 N_{1}+1=93 \mathrm{D}$ sigma-simulations are required, which are drawn at the center and within the principal axis of the multivariate distribution (two simulations per axis at $+/$ - standard deviation of the axis from the mean).

Then we calculate the scores of these $\mathrm{m}$ surrogate simulations $Y_{j}=\phi\left(X_{j}\right)$ and provide the scores to the CMA-ES algorithm "as if" they were the scores of the $\mathrm{m} 3 \mathrm{D}$ simulations. 
Table 4: Features and parameters in the 3D model personalisation to a real volume curve

\begin{tabular}{l|l}
\hline Features & 3D Parameters \\
\hline Maximal Volume Vmax & Maximal Contraction $\sigma$ \\
Stroke Volume $\mathbf{S V}$ & Stiffness $\mathbf{c 1}$ \\
Percentage of filling when the atrium contracts $\mathbf{F V a t}$ & Resting Mesh $\mathbf{R M}$ \\
Flow when the atrium contracts $\mathbf{Q a t}$ & 3D damping $\mu$ \\
Time where the volume is minimum $\mathbf{t V m i n}$ & \\
\hline
\end{tabular}

\subsection{Results}

In order to compare the efficiency of the Coupled-CMA optimization, we compare it with the full CMA optimization with a population size of 80 (CMA-80), as well as with a direct CMA optimization with a population size of 9 (CMA-9) which has the same computational burden than the Coupled-CMA.

This is done by comparing the scores of the $3 \mathrm{D}$ simulations made with the algorithm's internal mean Im at each iteration, which is the algorithm's current best estimate of the minimal parameters (In the case of Coupled-CMA, it is the score of the central sigma-simulation). Results are shown in Figure 5 (left).

The CMA-80 expectedly converges a lot faster than the CMA-9, but the main result is that the Coupled-CMA optimization converges at the same speed than the CMA-80. From a computing viewpoint, the Coupled-CMA lowers the number of 3D simulations from 80 to 9 at each iteration while keeping the accuracy, thus saving between 50 and 150 hours of CPU time at each iteration.

This similarity of convergence between CMA-80 and Coupled-CMA was confirmed on other geometries and values to fit, and overall we applied this method to the personalisation of 34 patient hearts, sucessfully matching the features to their target clinical values, within $6 \%$ of the mean value of each feature in the population, for at least $90 \%$ of the cases, as show on Figure 5 (right).
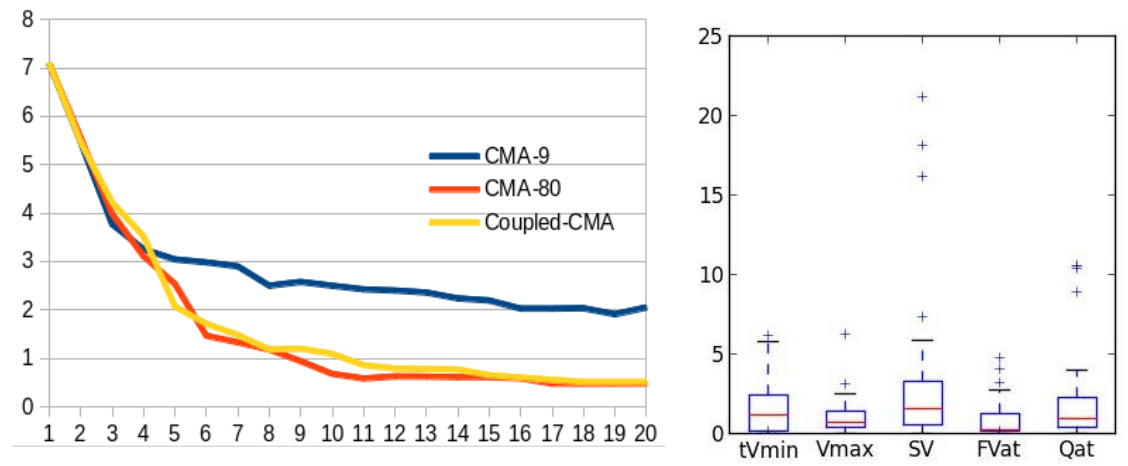

Fig. 5: Left: Score per iteration for the 3D methods. Right: Fitting error of each feature for the 34 patients (in percentage of the mean observed value of each feature) 


\section{Conclusion}

In this manuscript we detailed how a fast reduced " $0 \mathrm{D}$ " model relying on the same equations but simplifying assumptions can be coupled to the original 3D model to approximate computationally-costly $3 \mathrm{D}$ simulations with fast $0 \mathrm{D}$ simulations within a domain of 3D parameters. For this we developed a novel method to learn a mapping between the parameters of 3D sigma-simulations within the domain, and the parameters of corresponding 0D simulations with the same mechanical behaviour. In learning how to go from 3D parameters to 0D parameters, we learned the difference between the 0D (lumped) hypothesis and the 3D hypothesis. We then used the coupled model to build a fast personalisation agent, by substituting many $3 \mathrm{D}$ simulations in the optimization process by their $0 \mathrm{D}$ counterparts. This enabled us to benefit from the higher speed of convergence while considerably reducing the computational burden.

Several aspects of this method can be directly extended to more (nonlinear) features and parameters, such as the pressure, heart rate and the boundary conditions. We could also improve the computational gain by reusing the mapping from one iteration of the CMA-ES to the next one when the domains are similar enough instead of recomputing it. Finally, we expect the personalisation method to scale well to more complex optimization problems with a larger parameter space, since CMA-ES is well suited for those problems.

Ackowledgements This work has been partially funded by the EU FP7-funded project MD-Paedigree (Grant Agreement 600932) and contributes to the objectives of the ERC advanced grant MedYMA (2011-291080).

\section{References}

1. Neumann, D. et al: Robust Image-Based Estimation of Cardiac Tissue Parameters and Their Uncertainty from Noisy Data. In MICCAI 2014, 9-16

2. Konukoglu, E. et al: Efficient probabilistic model personalization integrating uncertainty on data and parameters: Application to eikonal-diffusion models in cardiac electrophysiology. Prog. Biophys. Mol. Biol. 107(1) (2011) 134-146

3. Esmaily, M. et al: A modular numerical method for implicit $0 \mathrm{~d} / 3 \mathrm{~d}$ coupling in cardiovascular finite element simulations. J. Comput. Phys. 244 (July 2013) 63-79

4. Chapelle, D. et al: Energy-preserving muscle tissue model: formulation and compatible discretizations. Int. J. Multiscale Comput. Eng., 10(2) (2012)

5. Marchesseau, S. et al: Fast parameter calibration of a cardiac electromechanical model from medical images based on the unscented transform. Biomech. model. in mechanobiol., 12(4), 815-831 (2013)

6. Caruel, M. et al: Dimensional reductions of a cardiac model for effective validation and calibration. Biomech. Model. Mechanobiol. 13(4) (2014) 897-914

7. Garny, A., Hunter, P.J.: Opencor: a modular and interoperable approach to computational biology. Frontiers in Physiology 6(26) (2015)

8. Hansen, N.: The cma evolution strategy: a comparing review. In: Towards a new evolutionary computation. Springer Berlin Heidelberg (2006) 75-102 